\title{
What makes the heart rhythm so intricate?
}

\author{
Jan P. Kucera \\ Department of Physiology \\ University of Bern \\ Bühlplatz 5 \\ CH-3012 Bern \\ Switzerland \\ $\begin{array}{ll}\text { Phone: } & +41316318759 \\ \text { Fax: } & \quad+41316314611 \\ \text { E-mail: } & \text { kucera@pyl.unibe.ch }\end{array}$
}

Accepted manuscript, published with minor editorial corrections

Heart Rhythm, 2014 Jul; 11(7):1220-1221

doi: 10.1016/j.hrthm.2014.04.013

PMID: 24727583

The analysis of heart rate variability (HRV) is routinely used to evaluate physiological regulatory mechanisms in diverse cardiac and non-cardiac conditions. In particular, the analysis of the typical high frequency and low frequency spectral peaks of HRV (around 0.25 and $0.1 \mathrm{~Hz}$ in humans) provides information regarding the function of the autonomic nervous system. ${ }^{1}$

One aspect that is still incompletely understood is the power law behavior of HRV in the very low frequency range. This power law is revealed by the linear aspect of the HRV power spectrum in a double logarithmic plot. The slope of the regression line in the double logarithmic plot provides the power law exponent, $\beta$. Processes exhibiting a power law behavior of their power spectrum appear statistically self-similar at all observation scales, a property which is typical of fractals.

The clinical importance of the fractal behavior of HRV was recognized in the 1990's in large studies that correlated $\beta$ with disease status and showed the potential prognostic value of this marker. For example, Bigger et al. $^{2}$ showed that $\beta$ lies around -1.2 in myocardial infarction patients and around -2 after cardiac transplantation, compared to -1 in control subjects; they also showed that more negative values of $\beta$ in myocardial infarction patients are related with a higher mortality.

It was postulated that the fractal behavior of $\mathrm{HRV}$ is due to interactions between multiple regulatory systems operating over a wide range of temporal scales, reflecting the ability of the organism to accommodate to stress. ${ }^{3}$ However, a power law behavior of beat rate variability (BRV) was also observed in cardiac systems devoid of any autonomic, endocrine or hemodynamic influences, such as cultures of neonatal rat ventricular myocytes, ${ }^{4}$ isolated rat cardiomyocytes, ${ }^{5}$ and even cultures of cardiomyocytes derived from human embryonic and induced pluripotent stem cells. ${ }^{6}$ Therefore, this power law behavior appears to be a universal phenomenon intrinsic to any type of cardiac cell or tissue. However, the underlying physiological mechanisms are still not fully established.

Nonlinear dynamics and chaos theory have fostered the development of various methods and markers to characterize the complexity of time series. One such marker is approximate entropy 
(ApEn), ${ }^{7}$ which quantifies regularity vs. complexity (i.e., predictability vs. unpredictability). However, studies of dynamical systems also revealed that power laws and fractal patterns can arise even in quite simple systems, such as the sandpile model of Bak et al., ${ }^{8}$ who proposed that such patterns emerge once a critical threshold of self-organization is reached. It must be noted that fractal markers such as $\beta$ are not necessarily related to complexity markers such as ApEn: a pattern doesn't need to be fractal to be complex, and a pattern doesn't need to be complex to be fractal.

In the study of Yaniv et al. ${ }^{9}$ published in this issue of Heart Rhythm, fractal measures of HRV and ApEn were investigated using a multi-scale approach from the whole organism to the isolated sinoatrial node (SAN) cell. The authors analyzed HRV in sedated rabbits and in animals in which the heart was denervated surgically and pharmacologically. They subsequently analyzed BRV in SAN tissue preparations isolated from these animals and in single dissociated SAN cells. Finally, they examined the effects of adrenergic and cholinergic stimulation of these cells.

In the heart in vivo, in the denervated heart and in SAN tissue, HRV and BRV exhibited a power law behavior with $\beta$ of $-1,-1.2$ and -1.6 , respectively. First, this observation confirms that autonomic influences modulate the power law behavior of HRV. Second, it confirms that this behavior is present in tissue devoid of any extrinsic influences. But most interestingly, the authors observed that the fractal behavior of BRV was present in only a small fraction of the isolated SAN cells. This indicates that intercellular interactions in the SAN promote the development of fractal BRV patterns. One interesting explanation proposed by the authors is the formation of cellular "neighborhoods" that synchronize their electrical activity and drive other neighborhoods and the rest of the SAN. Extending this line of thought, one can envision neighborhoods of neighborhoods, neighborhoods of neighborhoods of neighborhoods, and so on, which leads to the conjecture that such a successive embedding at different spatial scales may contribute to the fractal nature of BRV and HRV. However, the study of Yaniv et al. also demonstrates that intercellular interactions are not a prerequisite for the development of fractal BRV patterns. Therefore, if fractal patterns require the existence of spatial interactions, one needs to search for such interactions within the single cardiomyocyte.

In previous work, Lakatta et al. established the " $\mathrm{Ca}^{2+}$ clock" as a pacemaking mechanism that parallels the classical "membrane clock" ${ }^{10}$ The $\mathrm{Ca}^{2+}$ clock is based on spontaneous diastolic stochastic $\mathrm{Ca}^{2+}$ release events from the sarcoplasmic reticulum, which enhance depolarization via the $\mathrm{Na}^{+} / \mathrm{Ca}^{2+}$ exchange current. These $\mathrm{Ca}^{2+}$ release events are potentiated by $\beta$-adrenergic stimulation. ${ }^{10}$ Because every myocyte contains thousands of interacting $\mathrm{Ca}^{2+}$ release units, the question whether spatial interactions between $\mathrm{Ca}^{2+}$ release units within a single cell may contribute to fractal BRV patterns is therefore pertinent. This hypothesis is supported by the recent study of Nivala et al., ${ }^{11}$ who investigated $\mathrm{Ca}^{2+}$ release patterns emerging in a threedimensional model of the myocyte comporting 20000 interacting $\mathrm{Ca}^{2+}$ release units. When extracellular $\mathrm{Ca}^{2+}$ was increased, isolated random release events coalesced to form larger and larger sparks, which eventually merged to form self-organized spatial waves and temporal oscillations. Importantly, in critical regimes, the distribution of the number of $\mathrm{Ca}^{2+}$ release units involved in a release event was described by a power-law behavior, which reflected selforganized criticality. This self-organization may then translate into a power law behavior of interbeat intervals and contribute to the generation of fractal BRV and HRV patterns. The hypothesis of self-organized criticality within a single cell is further supported by the observation 
of Yaniv et al. that isoproterenol significantly increased the proportion of SAN cells exhibiting a fractal BRV pattern.

In conclusion, the power law behavior of HRV is likely to be the manifestation of universal, selforganized multi-scale phenomena spanning the entire range from the macromolecular complex to the entire organism. In the future, we need to understand how this power law behavior is affected quantitatively by various conditions, which will hopefully provide more refined markers utilizable for risk stratification, diagnosis and prognosis during heart disease. One approach would be to combine different markers, such as $\beta$ and ApEn. In the experiments of Yaniv et al., ApEn was smaller in isolated SAN tissue than in the heart in situ. This suggests that beat rate complexity is increased by the autonomic nervous system and possibly by further extracardiac mechanisms. However, the largest ApEn was observed in isolated SAN cells, suggesting that mutual synchronization of SAN cells within the SAN causes a decrease in complexity. These are very interesting findings, but their exact physiological signification remains a mystery which will have to be elucidated in further work.

\section{References}

1. Camm AJ, Malik M. Task force of the european society of cardiology and the north american society of pacing and electrophysiology: Heart rate variability: Standards of measurement, physiological interpretation, and clinical use. Eur Heart J. 1996;17:354-381.

2. Bigger JT, Steinman RC, Rolnitzky LM, Fleiss JL, Albrecht P, Cohen RJ. Power law behavior of RR-interval variability in healthy middle-aged persons, patients with recent acute myocardial infarction, and patients with heart transplants. Circulation. 1996;93:2142-2151.

3. Iyengar N, Peng CK, Morin R, Goldberger AL, Lipsitz LA. Age-related alterations in the fractal scaling of cardiac interbeat interval dynamics. Am J Physiol. 1996;271:R1078-1084.

4. Kucera JP, Heuschkel MO, Renaud P, Rohr S. Power-law behavior of beat-rate variability in monolayer cultures of neonatal rat ventricular myocytes. Circ Res. 2000;86:1140-1145.

5. Harada T, Yokogawa T, Miyaguchi T, Kori H. Singular behavior of slow dynamics of single excitable cells. Biophys J. 2009;96:255-267.

6. Mandel Y, Weissman A, Schick R, Barad L, Novak A, Meiry G, Goldberg S, Lorber A, Rosen MR, Itskovitz-Eldor J, Binah O. Human embryonic and induced pluripotent stem cell-derived cardiomyocytes exhibit beat rate variability and power-law behavior. Circulation. 2012;125:883-893.

7. Pincus SM. Approximate entropy as a measure of system complexity. Proc Natl Acad Sci USA. 1991;88:2297-2301.

8. Bak P, Tang C, Wiesenfeld K. Self-organized criticality: An explanation of the $1 / \mathrm{f}$ noise. Phys Rev Lett. 1987;59:381-384.

9. Yaniv Y, Ahmet I, Liu J, Lyashkov AE, Guiriba TR, Okamoto Y, Ziman BD, Lakatta EG. Synchronization of sinoatrial node pacemaker cell clocks and its autonomic modulation impart complexity to heart beating intervals. Heart Rhythm. 2014:in press.

10. Lakatta EG, Vinogradova T, Lyashkov A, Sirenko S, Zhu W, Ruknudin A, Maltsev VA. The integration of spontaneous intracellular $\mathrm{Ca}^{2+}$ cycling and surface membrane ion channel activation entrains normal automaticity in cells of the heart's pacemaker. Ann NY Acad Sci. 2006;1080:178206.

11. Nivala M, Ko CY, Weiss JN, Qu Z. Criticality in intracellular calcium signaling in cardiac myocytes. Biophys J. 2012;102:2433-2442. 